\title{
Development of Measuring System for the Non-Repetitive Run-Out(NRRO) of Ball Bearing
}

\author{
Y. Chen ${ }^{1, a, *}$, G.F. Hu ${ }^{1, b}$, X.S. Zhao ${ }^{1, c}$, D.W. Zhang ${ }^{1, d}$ \\ ${ }^{1}$ School of Mechanical Engineering, Tianjin University, Weijin Road, Tianjin, China \\ a chenye@tju.edu.cn, ${ }^{b}$ gaofenghu@tju.edu.cn, ${ }^{\mathrm{c}} 13521581683 @ 139 . c o m,{ }^{\mathrm{d}}$ medzhang@tju.edu.cn \\ *Corresponding author
}

Keywords: Angular Contact Ball Bearing, NRRO, Hydrostatic Spindle, Error Motion.

\begin{abstract}
The non-repetitive run-out(NRRO) of ball bearing critically influences the rotational accuracy of a machine tool. The quality of manufacturing can be improved by reducing the value of NRRO. This article developed a new ball bearing measuring system to investigate the NRRO caused geometrical errors of the bearing parts include inner and outer races and balls. The characteristic of the proposed measuring system as follows:(1) A high-precision hydrostatic spindle is applied to drive the inner race of the test bearing to obtain the system accuracy; (2) Measurable and variable axial preload is adopted to simulate the operating condition of bearings; (3) A 2 degrees of freedom flexure hinge mechanism (2-DOF FHM) is used to prevent the outer race from rotating; (4) Two capacitive sensors are used to measure radial error of the test bearing to ensure nano resolution.
\end{abstract}

\section{Introduction}

Rolling element bearing is the most common part used to support the rotational shaft, the rotational accuracy of which influences the performance of the mechanical system [1]. With the requirement of high precision and high speed, the dynamical performance of bearings need to be improved. The corresponding measuring system is developed to investigate the performance of angular contact ball bearings.

Spindle rotation error motions for machine tool directly affect the profile and the surface roughness of the work pieces directly. On the basis of the relationship with the rotation frequency, the error motion can be classified into synchronous and asynchronous error motions [2]. Synchronous error motion is the portion of total error motion that occurs at integer multiples of the rotation frequency (fundamental frequency). Asynchronous error motion is the portion of total error motion that occurs at frequencies other than integer multiples of the rotation frequency. The error motion of the asynchronous components is known as Non-Repetitive Run-Out(NRRO), which mainly affects the surface roughness of the work-piece. 
For the past years, some efforts have been made in investigating the NRRO of ball bearings. Jang et al. investigates the source of NRRO by analyzing the NRRO of the outer-race rotating spindle and the inner-race rotating spindle [3]. They reduce NRRO by inserting the visco-elastic material in the transmission path of NRRO. Noguchi and Kanada propose a measuring system for measuring radial NRRO [4]. In the measuring system, the axial preload is $100 \mathrm{~N}$ at maximum. Eric et al. develops a bearing measuring system by using air bearing reference spindle [5]. However, they ignore the influence of the torque arm on the radial error motion. Yang et al. develop a bearing analyzer by applying non-contact preload force, but they ignore the influence of the friction torque inside the test bearing [6]. This paper aims to develop a measuring system to investigate NRRO of ball bearings by simulating the operating condition, including different axial preload forces and rotational speeds.

\section{Measuring system}

The proposed measuring system in this paper is designed in accordance with ASME B89.3.42010 (Axes of rotation: methods for specifying and testing) and ISO 1132-2: 2001 (Rolling bearings - Tolerances - Part 2: Measuring and gauging principles and methods) [2,7]. A schematic view of the monolithic measuring system for radial NRRO is shown in Figure 1.

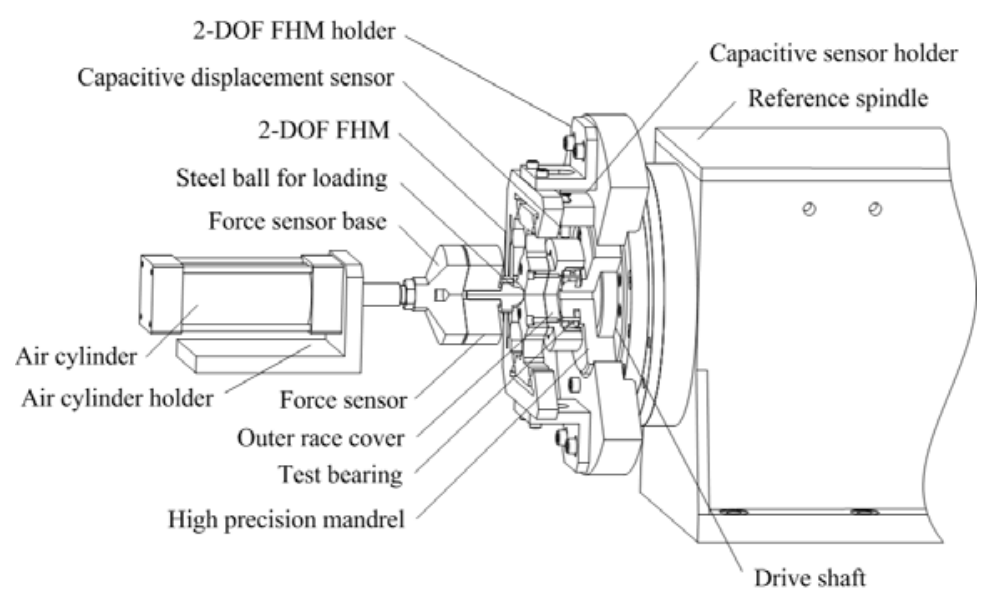

Figure 1 The general schematic view of the measuring system

The characteristics of the proposed measuring system are as follows:

(1) Drive module: a high-precision hydrostatic bearing and servo motor are applied to rotate the inner race if the test bearing for obtaining the required rotational accuracy and speed.

(2) Preloading module: an air cylinder is used to provide axial preloading force which can be measured by the force sensor.

(3) Supporting module: a 2 degrees of freedom flexure hinge mechanism (2-DOF FHM) is used to support the test bearing for preventing the outer race from rotating. The influence of the 2-DOF FHM are investigated to improve the radial NRRO measurement.

(4) Measuring module: two capacitive sensors with nano-level resolution are used to measure radial run-out of the test bearing. Displacement data are acquired by the data acquisition(DAQ) software running in the computer for further processing.

The experimental setup of the system for measuring the radial NRRO is shown in Figure 2. The radial error of the reference spindle is no more than $300 \mathrm{~nm}$. For ball bearings, the radial error can be treated as ignorable when processing the displacement data. The axial preloading force can be controlled form 0 to $1000 \mathrm{~N}$. The large range of preload can simulate operating condition of the test bearing. The orientation angles of the spindle is recorded by an optical index sensor in order to 
correspond to the displacement data. The acquired displacement data can be plotted by the form of polar plot.

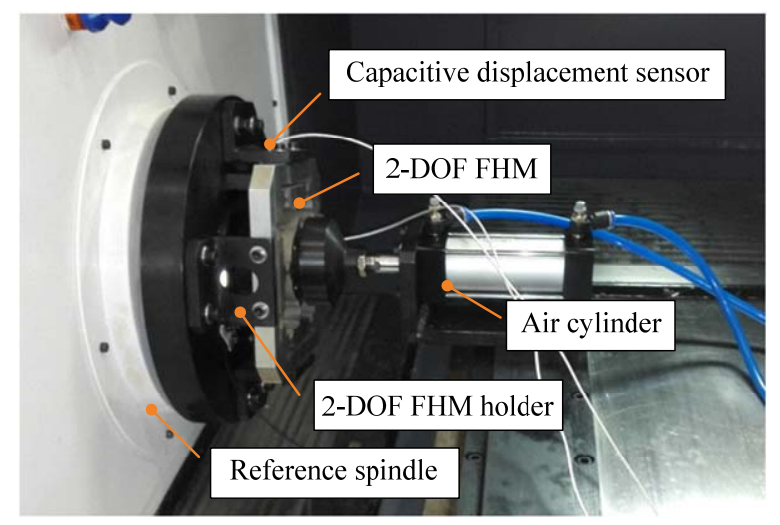

Figure 2 Experimental setup of the measuring system

Table 1 shows the measuring conditions in this research. The experiment is conducted in the invariable temperature environment. The thermal error generated inside the test bearing can be treated as ignorable. An ball bearing $7010 \mathrm{C} / \mathrm{P} 5$ is chosen as the test bearing in the experiment.

Table 1 Measuring conditions

\begin{tabular}{|l|l|}
\hline Axial preload(N) & $75,150,390,780$ \\
\hline Rotational speed(RPM) & $100,200,1200,2400,3000,4000$ \\
\hline Environment temperature & $20^{\circ} \mathrm{C} \pm 1^{\circ} \mathrm{C}$ \\
\hline
\end{tabular}

\section{Measurement results for current bearing}

Figure 3 shows the overwritten polar plot of the test bearing by using the radial displacement data. The displacement data include the synchronous and asynchronous components. The asynchronous components can be separated from the synchronous components according to frequency character. The width of the overwritten polar plot is evaluated as NRRO value.

The overwritten polar plots of the radial NRRO of the test bearing by removing the synchronous components at different rotational speeds are shown in Figure 4. The value of NRRO can be evaluated in accordance with ASME B89.3.4-2010 and ISO 1132-2: 2001. The value varies from $0.30 \mu \mathrm{m}$ to $0.98 \mu \mathrm{m}$ as the rotational speed changes at the same preloading force.

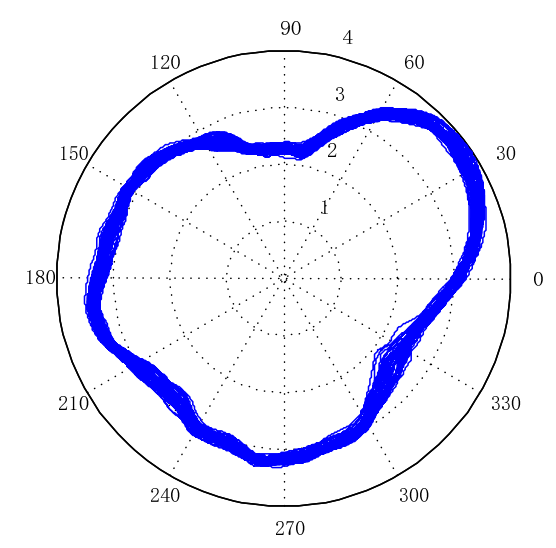

Figure 3 The overwritten radial polar plot of the test bearing(preload is $390 \mathrm{~N}$ and rotational speed is 200RPM) 

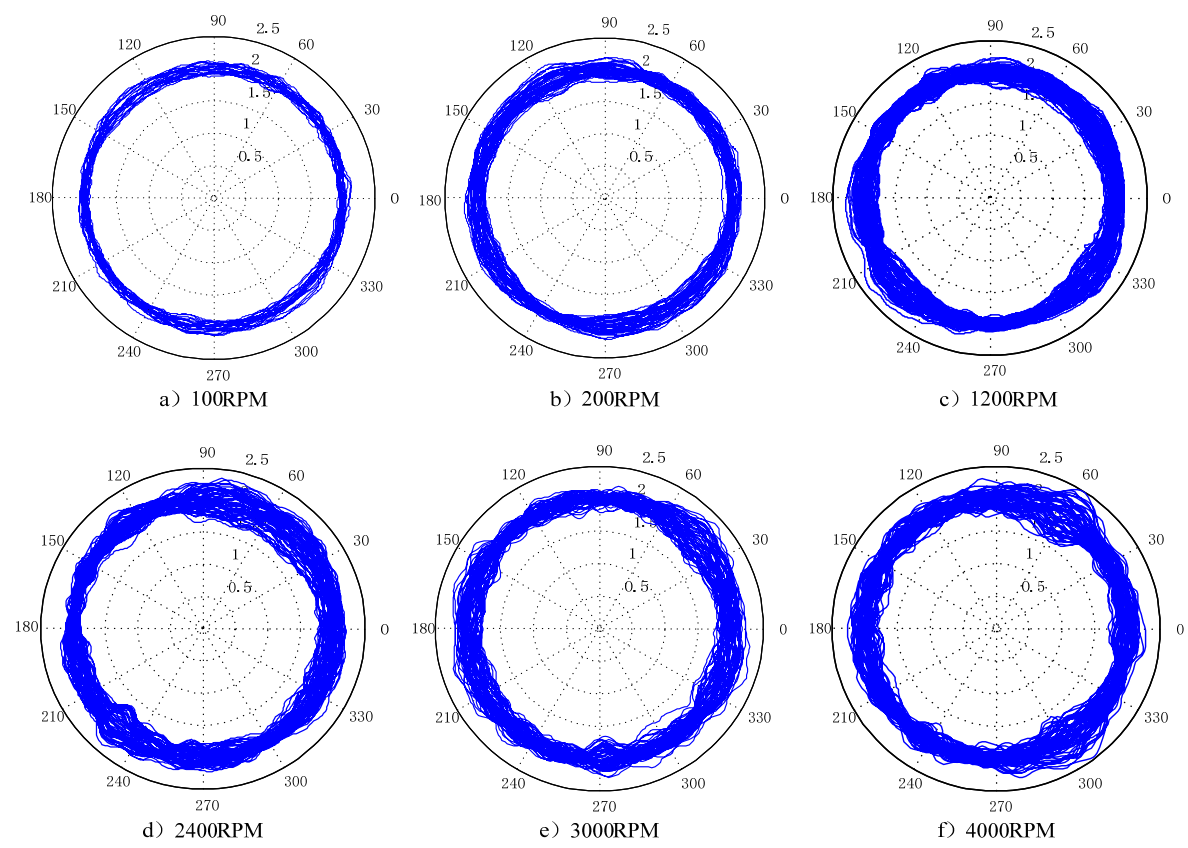

Figure 4 Polar plots of the radial NRRO of the test bearing at different rotational speeds

The polar plots of the radial NRRO of the test bearing are shown in Figure 5 at different preloading force when the rotational speed(1200RPM) is fixed. The value of NRRO becomes smaller when larger preloading force applying.
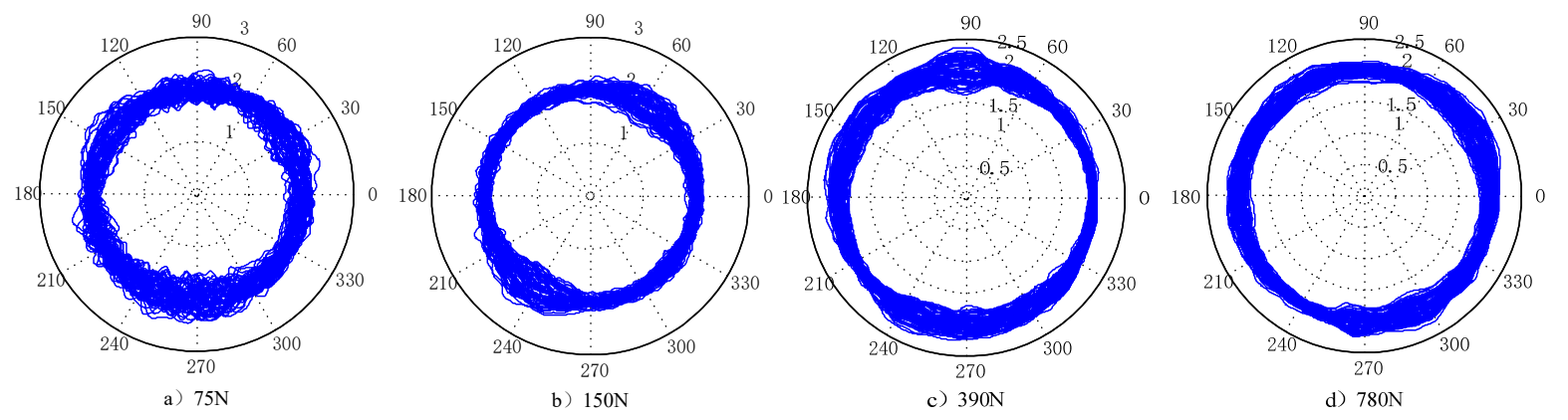

Figure 5 Polar plots of the radial NRRO of the test bearing at different preloading force

Table 2 Evaluated values for radial NRRO (unit: $\mu \mathrm{m}$ )

\begin{tabular}{|c|c|c|c|c|}
\hline & \multicolumn{4}{|c|}{ Axial preload (N) } \\
\hline Rotational speed(RPM) & 75 & 150 & 390 & 780 \\
\hline 100 & 0.91 & 0.49 & 0.30 & 0.33 \\
\hline 200 & 0.86 & 0.62 & 0.59 & 0.47 \\
\hline 1200 & 1.07 & 0.87 & 0.73 & 0.66 \\
\hline 2400 & 1.28 & 1.07 & 0.78 & 0.74 \\
\hline 3000 & 1.98 & 1.22 & 0.82 & 0.79 \\
\hline 4000 & 1.99 & 1.17 & 0.98 & 0.87 \\
\hline
\end{tabular}

Table 2 lists the corresponding radial NRRO values at different rotational speed and preloading force. The results indicate that the radial NRRO increase as the speed increases for the same axial preloading force. However, for the same rotational speed, NRRO decrease as the axial preloading force increases. The results of the radial NRRO of the test bearing include the error motion inside 
and outside the test bearing. The measured NRRO is the combined result. The way combined theory and experiment are used to study the reason of NRRO by the consideration of waviness of inner and outer ring raceways and ball.

\section{Conclusions}

This paper develops a measuring system for the non-repetitive run-out (NRRO) of ball bearings. The system mainly consists of high-precision and high-stiffness reference spindle, 2 degrees of freedom flexure hinge mechanism (2-DOF FHM), air cylinder, nano-resolution displacement sensors and data acquisition (DAQ) software and so on. By using the proposed measuring system, a serial of experiments are carried out to investigate the radial NRRO as the rotational speed and preloading force change. The experimental results indicate that the radial NRRO varies significantly in terms of the variation of the rotational speed and the preloading force. The results demonstrate that the proposed measuring system for the radial NRRO of ball bearing is feasible and has good repeatability, which can be used to investigate the dynamical character of ball bearing.

In further study, the lager preloading force will be applied and the measuring system will be more universal and flexible. Both radial and axial error motions can be measured at different rotational speeds and axial preloads.

\section{Acknowledgements}

This work was supported by the National Science and Technology Major Project of China under Grant No. 2014ZX04011011 and No.2015ZX04005001.

\section{References}

[1] D. L. Martin, A. N. Tabenkin, F. G. Parsons. (1995) Precision spindle and bearing error analysis. International Journal of Machine Tool \& Manufacture, 2(35), 187-193.

[2] ASME B89.3.4-2010 Axes of rotation: methods for specifying and testing.

[3] G. H. Jang, Member, IEEE, D. K. Kim, and J. H. Han. (2001) Characterization of NRRO in a HDD Spindle System Due to Ball Bearing Excitation, IEEE TRANSACTION ON MAGNETICS, 37, 815-819.

[4] S. Noguchi, T. Kanada. (2008) Development of measuring system for radial non-repetitive run-out (NRRO) and perception about present state of angular contact ball bearing for machine tools, Tribology International, 41, 1176-1180.

[5] E.R. Marsh. (2010) Precision Spindle Metrology, DEStech Publications.

[6] Z. Yang, J. Hong, J. Zhang, M. Y. Wang, and Y. Zhu. (2013) Nano-level instrumentation for analyzing the dynamic accuracy of a rolling element bearing", Review of Scientific Instruments, 84, 125103.

[7] ISO 1132-2: 2001, Rolling bearings - Tolerances - Part 2: Measuring and gauging principles and methods. 